\title{
Facile Synthesis of New Polyazamacrocycles by the Pd-Catalyzed Amination of 3,3'-Dibromobiphenyl
}

\author{
Alexei D. Averin, ${ }^{a}{ }^{@}$ Alexei N. Uglov, ${ }^{a}$ Alexei K. Buryak, ${ }^{b}$ and Irina P. Beletskaya ${ }^{a, b}$ \\ ${ }^{a}$ Lomonosov Moscow State University, Department of Chemistry, Leninskie Gory, Moscow, 119991 Russia. \\ ${ }^{\mathrm{b}}$ A. N. Frumkin Institute of Physical and Electrochemistry, 31 Leninskii prosp., Moscow, 119991, Russia. \\ ${ }^{\circledR}$ Corresponding authorE-mail: averin@org.chem.msu.ru
}

\begin{abstract}
Pd-Catalyzed amination of 3,3'-dibromobiphenyl using various polyamines and oxadiamines was studied. Target macrocycles were obtained in yields from moderate to good with a variety of polyamines and oxadiamines, cyclic oligomers were isolated in the majority of cases as by-products. Synthesis of the macrocycles containing two 3,3'-disubstituted biphenyls and two polyamine fragments (cyclodimers) was elaborated using intermediate di(bromobiphenyl)substituted polaymines or bis(polyamine)substituted biphenyls.
\end{abstract}

Keywords: Macrocycles, amination, catalysis, polyamines, biphenyl.

\section{Introduction}

Macrocycles containing biphenyl units attract a constant interest of researchers due to interesting coordination possibilities arising from attaching flexible and tunable polyoxa- and polyazacycles to a rigid non-planar aryl moiety. The most of reported macrocycles based on biphenyls were synthesized using non-catalytic approaches. Cyclic polyethers were formed starting from 2,2'-dihydroxybiphenyl, ${ }^{[1-3]}$ and their coordination with cations like tert-butylammonium was studied. ${ }^{[2]}$ Transport of $\mathrm{Li}, \mathrm{Na}, \mathrm{K}$ cations ${ }^{[4,5]}$ and of $\mathrm{Hg}\left(\mathrm{CF}_{3}\right)_{2}{ }^{[6,7]}$ through a liquid membrane was investigated using macrocycles of similar structure, in which one or two polyoxaethylene chains were attached to one biphenyl unit. Polyoxadiaminomacrocycles were also synthesized on the basis of 2,2'-disubstituted biphenyl and their complexation of primary alkylammonium salts, including chiral ones, was studied. ${ }^{[8]}$ Polyazamacrocycles with 3, 4 and 8 nitrogen atoms were investigated as complexing agents for $\mathrm{Cu}^{2+}, \mathrm{Zn}^{2+}$ and $\left[\mathrm{PdCl}_{4}\right]^{2-}$ ions. ${ }^{[9]}$ More sophisticated macrocycles like peptide-biphenyl hybrid ${ }^{[10]}$ and hemispherand macrocycle ${ }^{[11]}$ with bi- and quaterphenyl moieites have been recently reported. Cyclic triamides ${ }^{[12]}$ as well as cyclic Schiff bases (trianglimines) ${ }^{[13,14]}$ comprise three 3,3'-disubstituted biphenyls, the latter can be aslo built on the basis of 4,4'-disubstituted biphenyls. In some cases biphenyl fragment was built using Pd-catalyzed coupling of two benzene moieties at the step of macrocyclization, as it was in the case of the compound with diazacrown, dipeptide and biphenyl fragments. ${ }^{[15]}$ It is to be mentioned that biphenyls are incorporated in some biologically active macrocycles, e.g. tricyclic glucopeptides of vancomicine group. ${ }^{[16]}$ To the moment, there are no literature data on the synthesis of biphenyl-based macrocycles which employs catalytic bond formation between aromatic and aliphatic parts of the molecule. In recent years we have accumulated experience on the application of the Buchwald-Hartwig amination ${ }^{[17]}$ in the synthesis of polyazamacrocycles starting from various dihaloarenes ${ }^{[18-21]}$ and we investigated this approach for the construction of biphenyl-based macrocycles.

\section{Experimental}

NMR spectra were registered using Bruker Avance 400 spectrometer, MALDI-TOF spectra were obtained with Bruker Ultraflex spectrometer using 1,8,9-trihydroxyanthracene as matrix and PEGs as standards. 2-Bromonitrobenzene, oxadiamines, polyamines, $\quad 2,2^{\prime}$-bis(diphenylphosphino)-1,1'-binaphthalene (BINAP), 2-dimethylamino-2'-dicyclohexylphosphinobiphenyl (DavePHOS), sodium tert-butoxide were purchased from Aldrich and Acros and used without further purification, $\operatorname{Pd}(\mathrm{dba})_{2}$ was synthesized according to the method described earlier. ${ }^{[2]} 3,3^{2}$-Dibromobiphenyl was synthesized in 3 steps from 2-bromonitrobenzene according to the procedure. ${ }^{[23]}$ Dioxane was distilled over $\mathrm{NaOH}$ followed by the distillation over sodium under argon, dichloromethane and methanol were distilled.

Typical procedure for the synthesis of macrocycles 3 .

A two-neck flask equipped with a magnetic stirrer and a condenser, flushed with dry argon, was charged with 3,3'-dibromobiphenyl (1) ( $0.5 \mathrm{mmol}, 156 \mathrm{mg})$, absolute dioxane ( 25 $\mathrm{ml}), \mathrm{Pd}(\mathrm{dba})_{2}(24 \mathrm{mg}, 8 \mathrm{~mol} \%)$ and BINAP $(28 \mathrm{mg}, 9 \mathrm{~mol} \%)$. The mixture was stirred for $2 \mathrm{~min}$, then appropriate polyamine $2(0.5$ $\mathrm{mmol})$ and ${ }^{\mathrm{B} u O N a}(1.5 \mathrm{mmol})$ were added, and the reaction mixture was refluxed for $24 \mathrm{~h}$. After cooling to the ambient temperature and filtration of the precipitate dioxane was evaporated in vacuo and the residue was chromatographed on silica gel using a sequence of eluents: $\mathrm{CH}_{2} \mathrm{Cl}_{2}, \mathrm{CH}_{2} \mathrm{Cl}_{2}-\mathrm{MeOH}$ 500:1 - 3:1, $\mathrm{CH}_{2} \mathrm{Cl}_{2}-\mathrm{MeOH}$-aq. $\mathrm{NH}_{3}$ 100:20:1 - 10:4:1.

11,22,26-Tetraazapentacyclo[25.3.1.12,6. $\left.1^{12,16} \cdot 1^{17,21}\right]$ tetratriaconta-1(31),2(34),3,5,12(33), 13, 15,17(32), 18,20,27,29dodecaene, $4 \boldsymbol{a}(\mathrm{n}=1)$. Obtained from $37 \mathrm{mg}$ of propane-1,3-diamine 2a. Eluent: $\mathrm{CH}_{2} \mathrm{Cl}_{2}-\mathrm{MeOH}$ 200:1. Yield $27 \mathrm{mg}$ (24\%). Pale-yellow crystals, m.p. $224-225^{\circ} \mathrm{C}$ (decomp.) $m / z$ (MALDI-TOF) found: 448.2593. $\mathrm{C}_{30} \mathrm{H}_{32} \mathrm{~N}_{4}$ requires $448.2627\left[\mathrm{M}^{+}\right]$. ' $\mathrm{H}$ NMR $\left(\mathrm{CDCl}_{3}, 297\right.$ K) $\delta_{\mathrm{H}}$ ppm: $1.94\left(4 \mathrm{H}\right.$, quintet, $\left.{ }^{3} J=6.1 \mathrm{~Hz}\right), 3.37\left(8 \mathrm{H}, \mathrm{t},{ }^{3} J=6.1 \mathrm{~Hz}\right)$, 3.91 (4H, br.s), $6.60\left(4 \mathrm{H}, \mathrm{dd},{ }^{3} J=7.6 \mathrm{~Hz},{ }^{4} J=1.8 \mathrm{~Hz}\right), 6.78(4 \mathrm{H}$, br.s), $6.91\left(4 \mathrm{H}, \mathrm{d},{ }^{3} J=7.7 \mathrm{~Hz}\right), 7.21\left(4 \mathrm{H}, \mathrm{t},{ }^{3} J=7.9 \mathrm{~Hz}\right) .{ }^{13} \mathrm{C}$ NMR $\left(\mathrm{CDCl}_{3}, 297 \mathrm{~K}\right) \delta_{\mathrm{c}} \mathrm{ppm}: 28.6(2 \mathrm{C}), 42.4(4 \mathrm{C}), 111.6$ (4C), 112.1 (4C), 116.5 (4C), 129.6 (4C), 142.9 (4C), 148.3 (4C). 
$7,10,13,24,27,30-H e x a a z a p e n t a c y c l o\left[29 \cdot 3 \cdot 1 \cdot 1^{2,6} \cdot 1^{14,18} \cdot 1^{19,23}\right]$ octatriaconta-1(35), 2(38), 3, 5, 14(37), 15, 17, 19(36), 20,22,31,33dodecaene, $4 \boldsymbol{b}(\mathrm{n}=1)$. Obtained from $52 \mathrm{mg}$ of triamine $\mathbf{2 b}$. Eluent: $\mathrm{CH}_{2} \mathrm{Cl}_{2}-\mathrm{MeOH} / \mathrm{NH}_{3}$-aq 100:20:1. Yield $29 \mathrm{mg}$ (23\%). Pale-yellow glassy solid. Contains admixtures of cyclotrimer $\mathbf{4 b}(\mathrm{n}=2)$ and cyclotetramer $4 \mathbf{b} \quad(\mathrm{n}=3) . \mathrm{m} / z$ (MALDI-TOF) $507.39 \quad(\mathbf{4 b} \quad(\mathrm{n}=1)$ $\left.\left[(\mathrm{M}+\mathrm{H})^{+}\right]\right), 760.44\left(\mathbf{4 b}(\mathrm{n}=2)\left[(\mathrm{M}+\mathrm{H})^{+}\right]\right), 1012.72\left(\mathbf{4 b}(\mathrm{n}=3)\left[\mathrm{M}^{+}\right]\right)$. ${ }^{1} \mathrm{H}$ NMR $\left(\mathrm{CDCl}_{3}, 297 \mathrm{~K}\right) \delta_{\mathrm{H}}$ ppm: $2.85(8 \mathrm{H}, \mathrm{br} . \mathrm{s}), 3.12-3.30(8 \mathrm{H}$, m), 6.50-6.58 (4H, m), 6.82-6.94 (8H, m), 7.11-7.21 (4H, m), NH protons were not assigned. ${ }^{13} \mathrm{C} \mathrm{NMR}\left(\mathrm{CDCl}_{3}, 297 \mathrm{~K}\right) \delta_{\mathrm{c}}$ ppm: 43.0 (4C), 48.0 (4C), 111.7 (4C), 111.8 (4C), 116.4 (4C), 129.4 (4C), $142.3(4 \mathrm{C}), 148.4(4 \mathrm{C})$.

$7,11,15-$ Triazatricyclo [14.3.1.12,6] henicosa1(20),2(21),3,5,16,18-hexaene, 3c. Obtained from $66 \mathrm{mg}$ of triamine 2c. Eluent: $\mathrm{CH}_{2} \mathrm{Cl}_{2}-\mathrm{MeOH} 3: 1$. Yield $35 \mathrm{mg}(25 \%)$. Paleyellow glassy solid. $m / z$ (MALDI-TOF) found: $281.1930 . \mathrm{C}_{18} \mathrm{H}_{23} \mathrm{~N}_{3}$ requires $281.1892\left[\mathrm{M}^{+}\right] .{ }^{1} \mathrm{H}$ NMR $\left(\mathrm{CDCl}_{3}, 297 \mathrm{~K}\right) \delta_{\mathrm{H}} \mathrm{ppm:} 1.82$ (4H, quintet, $\left.{ }^{3} J=6.7 \mathrm{~Hz}\right), 2.71\left(4 \mathrm{H}, \mathrm{t},{ }^{3} J=6.0 \mathrm{~Hz}\right), 3.37\left(4 \mathrm{H}, \mathrm{t},{ }^{3} J\right.$ $=7.3 \mathrm{~Hz}), 4.06\left(2 \mathrm{H}\right.$, br.s), $6.54\left(2 \mathrm{H}, \mathrm{dd},{ }^{3} J=8.0 \mathrm{~Hz},{ }^{4} J=0.8 \mathrm{~Hz}\right)$, $7.03\left(2 \mathrm{H}, \mathrm{d},{ }^{3} J=7.5 \mathrm{~Hz}\right), 7.17\left(2 \mathrm{H}, \mathrm{t},{ }^{3} J=7.8 \mathrm{~Hz}\right), 7.29$ (2H, br.s), $\mathrm{NH}$ proton of the dialkylamino group was not assigned. ${ }^{13} \mathrm{C}$ NMR $\left(\mathrm{CDCl}_{3}, 297 \mathrm{~K}\right) \delta_{\mathrm{c}}$ ppm: 29.7 (2C), 41.3 (2C), 47.0 (2C), 109.6 (2C), $114.0(2 \mathrm{C}), 114.8(2 \mathrm{C}), 129.3(2 \mathrm{C}), 142.1(2 \mathrm{C}), 147.9(2 \mathrm{C})$.

$7,11,15,26,30,34-H e x a a z a p e n t a c y c l o\left[33 \cdot 3 \cdot 1 \cdot 1^{2,6} \cdot 1^{16,20} \cdot 1^{21,25}\right]$ dotetraconta-1(39), 2(42), 3, 5,16(41), 17,19,21(40), 22,24,35,37dodecaene, $4 c(n=1)$. Obtained as by-product in the synthesis of macrocycle 3c. Eluent: $\mathrm{CH}_{2} \mathrm{Cl}_{2}-\mathrm{MeOH}-\mathrm{NH}_{3}$-aq 100:20:1. Yield 30 $\mathrm{mg}(21 \%)$. Pale-yellow glassy solid. $\mathrm{m} / z$ (MALDI-TOF) found: 562.3701. $\mathrm{C}_{36} \mathrm{H}_{46} \mathrm{~N}_{6}$ requires $562.3784\left[\mathrm{M}^{+}\right] .{ }^{1} \mathrm{H} \mathrm{NMR}\left(\mathrm{CDCl}_{3}, 297\right.$ K) $\delta_{\mathrm{H}}$ ppm: $1.80\left(8 \mathrm{H}\right.$, quintet, $\left.{ }^{3} J=6.7 \mathrm{~Hz}\right), 2.68\left(8 \mathrm{H}, \mathrm{t},{ }^{3} J=5.9\right.$ $\mathrm{Hz}), 3.29\left(8 \mathrm{H}, \mathrm{t},{ }^{3} J=5.1 \mathrm{~Hz}\right), 3.90(4 \mathrm{H}, \mathrm{br} . \mathrm{s}), 6.53\left(4 \mathrm{H}, \mathrm{d},{ }^{3} J=8.0\right)$, $7.02\left(4 \mathrm{H}, \mathrm{d},{ }^{3} \mathrm{~J}=7.4 \mathrm{~Hz}\right), 7.16\left(4 \mathrm{H}, \mathrm{t},{ }^{3} \mathrm{~J}=7.7 \mathrm{~Hz}\right), 7.17$ (4H, br.s), $\mathrm{NH}$ proton of the dialkylamino group was not assigned. ${ }^{13} \mathrm{C} \mathrm{NMR}$ $\left(\mathrm{CDCl}_{3}, 297 \mathrm{~K}\right) \delta_{c} \mathrm{ppm}: 28.4(4 \mathrm{C}), 41.1(4 \mathrm{C}), 48.0(4 \mathrm{C}), 109.8(4 \mathrm{C})$, 114.0 (4C), 114.8 (4C), 129.3 (4C), 142.3 (4C), 147.7 (4C).

7,10,13,16-Tetraazatricyclo[15.3.1.1 $\left.1^{2,6}\right]$ docosa1(21),2(22),3,5,17,19-hexaene, 3d. Obtained from $73 \mathrm{mg}$ of tetraamine 2d. Eluent: $\mathrm{CH}_{2} \mathrm{Cl}_{2}-\mathrm{MeOH}-\mathrm{NH}_{3}$-aq 100:20:3. Yield 23 $\mathrm{mg}$ (16\%). Pale-yellow glassy solid. $\mathrm{m} / \mathrm{z}$ (MALDI-TOF) found: 296.1959. $\mathrm{C}_{18} \mathrm{H}_{24} \mathrm{~N}_{4}$ requires $296.2001\left[\mathrm{M}^{+}\right] .{ }^{1} \mathrm{H}$ NMR $\left(\mathrm{CDCl}_{3}, 297\right.$ K) $\delta_{\mathrm{H}} \mathrm{ppm}: 2.82(4 \mathrm{H}, \mathrm{s}), 2.87\left(4 \mathrm{H}, \mathrm{t},{ }^{3} J=6.6 \mathrm{~Hz}\right), 3.29\left(4 \mathrm{H}, \mathrm{t},{ }^{3} \mathrm{~J}=\right.$ $6.6 \mathrm{~Hz}), 6.54\left(2 \mathrm{H}, \mathrm{dd},{ }^{3} J=8.0 \mathrm{~Hz},{ }^{4} J=2.0 \mathrm{~Hz}\right), 7.02\left(2 \mathrm{H}, \mathrm{d},{ }^{3} J=7.7\right.$ $\mathrm{Hz}), 7.06(2 \mathrm{H}, \mathrm{br} . \mathrm{s}), 7.16\left(2 \mathrm{H}, \mathrm{t},{ }^{3} J=7.7 \mathrm{~Hz}\right), \mathrm{NH}$ protons were not assigned. ${ }^{13} \mathrm{C} \mathrm{NMR}\left(\mathrm{CDCl}_{3}, 297 \mathrm{~K}\right) \delta_{\mathrm{c}} \mathrm{ppm}: 44.27(2 \mathrm{C}), 48.5(2 \mathrm{C})$, 49.3 (2C), 108.9 (2C), 114.2 (2C), 115.7 (2C), 129.3 (2C), 142.2 (2C), $148.9(2 \mathrm{C})$

$7,10,13,16,27,30,33,36-$ Octaazapentacyclo[35.3.1.1 $1^{2,6} \cdot 1^{17,21}$. $\left.1^{22,26}\right]$ tetratetraconta-1(41),2(44), 3,5,17(43), 18,20,22(42), $23,25,37,39-$ dodecaene, $4 \boldsymbol{d}(\mathrm{n}=1)$. Obtained as by-product in the synthesis of macrocycle 3d. Eluent: $\mathrm{CH}_{2} \mathrm{Cl}_{2}-\mathrm{MeOH}-\mathrm{NH}_{3}$-aq 100:25:5. Yield $27 \mathrm{mg}$ (18\%). Pale-yellow glassy solid. Contains admixture of cyclotrimer $\mathbf{4 d}(\mathrm{n}=2) . \mathrm{m} / z$ (MALDI-TOF) 592.33 (4d $\left.(\mathrm{n}=2)\left[\mathrm{M}^{+}\right]\right), 888.38\left(\mathbf{4 d}(\mathrm{n}=2)\left[\mathrm{M}^{+}\right]\right) .{ }^{1} \mathrm{H}$ NMR $\left(\mathrm{CDCl}_{3}, 297 \mathrm{~K}\right) \delta_{\mathrm{H}}$ ppm: $2.74(8 \mathrm{H}, \mathrm{s}), 2.85\left(8 \mathrm{H}, \mathrm{t},{ }^{3} J=5.8 \mathrm{~Hz}\right), 3.21\left(8 \mathrm{H}, \mathrm{t},{ }^{3} J=5.2 \mathrm{~Hz}\right)$, $6.56\left(4 \mathrm{H}, \mathrm{dd},{ }^{3} J=7.8 \mathrm{~Hz},{ }^{4} J=1.9 \mathrm{~Hz}\right), 6.80(4 \mathrm{H}, \mathrm{s}), 6.89(4 \mathrm{H}, \mathrm{d}$, $\left.{ }^{3} J=7.2 \mathrm{~Hz}\right), 7.15\left(4 \mathrm{H}, \mathrm{t},{ }^{3} J=7.8 \mathrm{~Hz}\right)$, NH protons were not assigned. ${ }^{13} \mathrm{C} \mathrm{NMR}\left(\mathrm{CDCl}_{3}, 297 \mathrm{~K}\right) \delta_{\mathrm{c}} \mathrm{ppm}: 43.5$ (4C), $48.4(4 \mathrm{C}), 48.8(4 \mathrm{C})$, 111.7 (4C), 111.8 (4C), 116.6 (4C), 129.5 (4C), 142.8 (4C), 148.6 (4C).

7,10,14,17-Tetraazatricyclo[16.3.1.12,6]tricosa1(22),2(23),3,5,18,20-hexaene, 3e. Obtained from $80 \mathrm{mg}$ of tetraamine 2e. Eluent: $\mathrm{CH}_{2} \mathrm{Cl}_{2}-\mathrm{MeOH}-\mathrm{NH}_{3}$-aq 100:20:2. Yield 41 $\mathrm{mg}(26 \%)$. Pale-yellow glassy solid. $m / z$ (MALDI-TOF) found: 310.2190. $\mathrm{C}_{19} \mathrm{H}_{26} \mathrm{~N}_{4}$ requires $310.2157\left[\mathrm{M}^{+}\right] .{ }^{1} \mathrm{H} \mathrm{NMR}\left(\mathrm{CDCl}_{3}, 297\right.$ $\mathrm{K}) \delta_{\mathrm{H}}$ ppm: $1.67\left(2 \mathrm{H}\right.$, quintet, $\left.{ }^{3} J=6.4 \mathrm{~Hz}\right), 2.80\left(4 \mathrm{H}, \mathrm{t},{ }^{3} J=6.4\right.$ $\mathrm{Hz}), 2.83\left(4 \mathrm{H}, \mathrm{t},{ }^{3} \mathrm{~J}=6.9 \mathrm{~Hz}\right), 3.36\left(4 \mathrm{H}, \mathrm{t},{ }^{3} J=6.9 \mathrm{~Hz}\right), 4.10(2 \mathrm{H}$, br.s), $6.57\left(2 \mathrm{H}, \mathrm{dd},{ }^{3} J=8.0 \mathrm{~Hz},{ }^{4} J=1.4 \mathrm{~Hz}\right), 6.96-7.00(4 \mathrm{H}, \mathrm{m})$, $7.17\left(2 \mathrm{H}, \mathrm{t},{ }^{3} \mathrm{~J}=7.7 \mathrm{~Hz}\right), \mathrm{NH}$ protons of the dialkylamino groups were not assigned. ${ }^{13} \mathrm{C}$ NMR $\left(\mathrm{CDCl}_{3}, 297 \mathrm{~K}\right) \delta_{\mathrm{c}}$ ppm: $29.5(1 \mathrm{C})$, 44.3 (2C), 48.0 (2C), $49.2(2 \mathrm{C}), 110.1$ (2C), $114.2(2 \mathrm{C}), 116.1(2 \mathrm{C})$, $129.3(2 \mathrm{C}), 142.7(2 \mathrm{C}), 148.7(2 \mathrm{C})$.

$7,10,14,17,28,31,35,38$-Octaazapentacyclo[37.3.1.1.1.6. $\cdot 1^{18,22}$ $\left.1^{23,27}\right]$ hexatetraconta-1(43),2(46), 3, 5, 18(45), 19,21,23(44), 24,26, 39,41-dodecaene, $4 \boldsymbol{e}(\mathrm{n}=1)$. Obtained as by-product in the synthesis of macrocycle 3e. Eluent: $\mathrm{CH}_{2} \mathrm{Cl}_{2}-\mathrm{MeOH}-\mathrm{NH}_{3}$-aq 100:20:3. Yield $12 \mathrm{mg}(8 \%)$. Pale-yellow glassy solid. $\mathrm{m} / \mathrm{z}$ (MALDI-TOF) found: 620.4307. $\mathrm{C}_{38} \mathrm{H}_{52} \mathrm{~N}_{8}$ requires $620.4314\left[\mathrm{M}^{+}\right] .{ }^{1} \mathrm{H} \mathrm{NMR}\left(\mathrm{CDCl}_{3}, 297\right.$ K) $\delta_{\mathrm{H}}$ ppm: $1.69\left(4 \mathrm{H}\right.$, quintet, $\left.{ }^{3} J=5.6 \mathrm{~Hz}\right), 2.77\left(8 \mathrm{H}, \mathrm{t},{ }^{3} J=4.6\right.$ $\mathrm{Hz}), 2.83\left(8 \mathrm{H}, \mathrm{t},{ }^{3} J=6.4 \mathrm{~Hz}\right), 3.24(8 \mathrm{H}, \mathrm{br} . \mathrm{s}), 6.57\left(4 \mathrm{H}, \mathrm{d},{ }^{3} J=7.8\right.$ $\mathrm{Hz}), 6.72$ (4H, br.s), $6.85\left(4 \mathrm{H}, \mathrm{d},{ }^{3} J=7.6 \mathrm{~Hz}\right), 7.14\left(4 \mathrm{H}, \mathrm{t},{ }^{3} J=7.7\right.$ $\mathrm{Hz}), \mathrm{NH}$ protons were not assigned. ${ }^{13} \mathrm{C} \mathrm{NMR}\left(\mathrm{CDCl}_{3}, 297 \mathrm{~K}\right) \delta$ c ppm: $28.9-29.7(\mathrm{~m}, 2 \mathrm{C}), 42.6-43.0(\mathrm{~m}, 4 \mathrm{C}), 47.8-49.3(\mathrm{~m}, 8 \mathrm{C})$, 111.2-111.8 (m, 8C), 116.3+116.4 (4C), 129.5 (4C), 142.6 (4C), $148.4+148.5(4 \mathrm{C})$.

7,11,14,18-Tetraazatricyclo[17.3.1.1 $\left.1^{2,6}\right]$ tetracosa1(23),2(24),3,5,19,21-hexaene, $3 f$. Obtained from $87 \mathrm{mg}$ of tetraamine 2f. Eluent: $\mathrm{CH}_{2} \mathrm{Cl}_{2}-\mathrm{MeOH} 3: 1-\mathrm{CH}_{2} \mathrm{Cl}_{2}-\mathrm{MeOH}-\mathrm{NH}_{3}$-aq 100:20:2. Yield $71 \mathrm{mg}(44 \%)$. Pale-yellow crystals, m.p. $115-116^{\circ} \mathrm{C}$. $\mathrm{m} / z$ (MALDI-TOF) found: $324.2264 . \mathrm{C}_{20} \mathrm{H}_{28} \mathrm{~N}_{4}$ requires 324.2314 $\left[\mathrm{M}^{+}\right] .{ }^{1} \mathrm{H}$ NMR $\left(\mathrm{CDCl}_{3}, 297 \mathrm{~K}\right) \delta_{\mathrm{H}} \mathrm{ppm}: 1.92\left(4 \mathrm{H}\right.$, quintet, ${ }^{3} J=6.6$ $\mathrm{Hz}), 2.82\left(4 \mathrm{H}, \mathrm{t},{ }^{3} J=6.0 \mathrm{~Hz}\right), 2.88(4 \mathrm{H}, \mathrm{s}), 3.32\left(4 \mathrm{H}, \mathrm{t},{ }^{3} J=7.2\right.$ Hz), 3.72 (2H, br.s), $6.53\left(2 \mathrm{H}, \mathrm{d},{ }^{3} J=7.6 \mathrm{~Hz}\right), 6.93(2 \mathrm{H}, \mathrm{s}), 6.94$ $\left(2 \mathrm{H}, \mathrm{d},{ }^{3} J=7.8 \mathrm{~Hz}\right), 7.15\left(2 \mathrm{H}, \mathrm{t},{ }^{3} J=7.7 \mathrm{~Hz}\right), \mathrm{NH}$ protons of the dialkylamino groups were not assigned. ${ }^{13} \mathrm{C} \mathrm{NMR}\left(\mathrm{CDCl}_{3}, 297 \mathrm{~K}\right)$

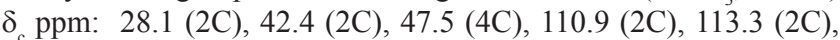
115.7 (2C), 129.5 (2C), 142.5 (2C), 148.3 (2C).

$7,11,14,18,29,33,36,40$-Octaazapentacyclo[39.3.1.1 $1^{2,6} \cdot 1^{19,23}$. $1^{24,28}$ Joctatetraconta-1(45), 2(48), 3, 5, 19(47), 20,22,24(46), 25,27, 41,43-dodecaene, $4 \boldsymbol{f}(\mathrm{n}=1)$. Obtained as by-product in the synthesis of macrocycle 3f. Eluent: $\mathrm{CH}_{2} \mathrm{Cl}_{2}-\mathrm{MeOH}-\mathrm{NH}_{3}$-aq 100:20:3. Yield $41 \mathrm{mg}(25 \%)$. Pale-yellow glassy solid. $\mathrm{m} / z$ (MALDI-TOF) found:

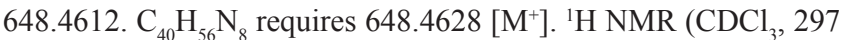
K) $\delta_{\mathrm{H}}$ ppm: $1.73\left(8 \mathrm{H}\right.$, quintet, $\left.{ }^{3} J=6.3 \mathrm{~Hz}\right), 2.71\left(8 \mathrm{H}, \mathrm{t},{ }^{3} \mathrm{~J}=6.3\right.$ $\mathrm{Hz}), 2.72(8 \mathrm{H}, \mathrm{s}), 3.15\left(8 \mathrm{H}, \mathrm{t},{ }^{3} J=6.3 \mathrm{~Hz}\right), 6.53\left(4 \mathrm{H}, \mathrm{dd},{ }^{3} J=7.8\right.$ $\left.\mathrm{Hz},{ }^{3} J=1.7 \mathrm{~Hz}\right), 6.76\left(4 \mathrm{H}, \mathrm{t},{ }^{4} J=1.7 \mathrm{~Hz}\right), 6.87\left(4 \mathrm{H}, \mathrm{d},{ }^{3} J=7.5 \mathrm{~Hz}\right)$, $7.17\left(4 \mathrm{H}, \mathrm{t},{ }^{3} \mathrm{~J}=7.7 \mathrm{~Hz}\right), \mathrm{NH}$ protons of the dialkylamino groups were not assigned. ${ }^{13} \mathrm{C} \mathrm{NMR}\left(\mathrm{CDCl}_{3}, 297 \mathrm{~K}\right) \delta$ ppm: $29.1(4 \mathrm{C})$, 42.9 (4C), 48.1 (4C), 48.9 (4C), 111.3 (4C), 111.9 (4C), 116.2 (4C), 129.3 (4C), $142.9(4 \mathrm{C}), 148.8$ (4C).

7,11,15,19-Tetraazatricyclo[18.3.1.12,6]pentacosa1(24),2(25),3,5,20,22-hexaene, 3g. Obtained from $94 \mathrm{mg}$ of tetraamine 2g. Eluent: $\mathrm{CH}_{2} \mathrm{Cl}_{2}-\mathrm{MeOH}-\mathrm{NH}_{3}$-aq 100:20:1. Yield $69 \mathrm{mg}$ (41\%). Pale-yellow glassy solid. $\mathrm{m} / \mathrm{z}$ (MALDI-TOF) found: 338.2410. $\mathrm{C}_{21} \mathrm{H}_{30} \mathrm{~N}_{4}$ requires $338.2470\left[\mathrm{M}^{+}\right]$. ${ }^{1} \mathrm{H}$ NMR $\left(\mathrm{CDCl}_{3}, 297\right.$ K) $\delta_{\mathrm{H}}$ ppm: $1.70\left(2 \mathrm{H}\right.$, quintet, $\left.{ }^{3} J=6.2 \mathrm{~Hz}\right), 1.83\left(4 \mathrm{H}\right.$, quintet, ${ }^{3} J=$ $6.6 \mathrm{~Hz}), 2.74\left(8 \mathrm{H}, \mathrm{t},{ }^{3} \mathrm{~J}=6.4 \mathrm{~Hz}\right), 3.22\left(4 \mathrm{H}, \mathrm{t},{ }^{3} J=6.9 \mathrm{~Hz}\right), 6.53(2 \mathrm{H}$, d, $\left.{ }^{3} J=8.0 \mathrm{~Hz}\right), 6.85(2 \mathrm{H}$, br.s $), 6.94\left(2 \mathrm{H}, \mathrm{d},{ }^{3} J=7.6 \mathrm{~Hz}\right), 7.19(2 \mathrm{H}$, $\left.\mathrm{t},{ }^{3} \mathrm{~J}=7.8 \mathrm{~Hz}\right), \mathrm{NH}$ protons were not assigned. ${ }^{13} \mathrm{C} \mathrm{NMR}\left(\mathrm{CDCl}_{3}\right.$, $297 \mathrm{~K}) \delta_{\mathrm{c}} \mathrm{ppm}: 28.5(2 \mathrm{C}), 29.8(1 \mathrm{C}), 43.1(2 \mathrm{C}), 48.4(2 \mathrm{C}), 48.5$ (2C), $110.7(2 \mathrm{C}), 112.2(2 \mathrm{C}), 115.4(2 \mathrm{C}), 129.2(2 \mathrm{C}), 142.5(2 \mathrm{C})$, $148.6(2 \mathrm{C})$.

$7,11,15,19,30,34,38,42$-Octaazapentacyclo[41.3.1.1 $1^{2,6}$ $\left.1^{20,24} \cdot 1^{25,29}\right]$ pentaconta-1(47),2(50),3,5,20(49),21,23,25(48), 26,28, 43,45-dodecaene, $\mathbf{4} \mathbf{g}(\mathrm{n}=1)$. Obtained as by-product in the synthesis of macrocycle 3g. Eluent: $\mathrm{CH}_{2} \mathrm{Cl}_{2}-\mathrm{MeOH}-\mathrm{NH}_{3}$-aq 100:4:1. Yield $32 \mathrm{mg}(19 \%)$. Contains admixtures of cyclotrimer $\mathbf{4 g}(\mathrm{n}=2)$ and cyclotetramer $4 \mathrm{~g}(\mathrm{n}=3)$. Pale-yellow glassy solid. $\mathrm{m} / \mathrm{z}$ (MALDITOF) $676.48\left(4 \mathrm{~g}(\mathrm{n}=1)\left[\mathrm{M}^{+}\right]\right), 1014.57\left(4 \mathrm{~g}(\mathrm{n}=2)\left[\mathrm{M}^{+}\right]\right), 1362.40$ $\left(4 \mathbf{g}(\mathrm{n}=3)\left[\mathrm{M}^{+}\right]\right) .{ }^{1} \mathrm{H}$ NMR $\left(\mathrm{CDCl}_{3}, 297 \mathrm{~K}\right) \delta_{\mathrm{H}}$ ppm: $1.72(12 \mathrm{H}, \mathrm{br} . \mathrm{s})$, 2.67 (16H, br.s), 3.11 (8H, br.s), $6.51\left(4 \mathrm{H}, \mathrm{d},{ }^{3} J=5.4 \mathrm{~Hz}\right), 6.76(4 \mathrm{H}$, br.s), $6.86\left(4 \mathrm{H}, \mathrm{d},{ }^{3} J=6.9 \mathrm{~Hz}\right), 7.15\left(4 \mathrm{H}, \mathrm{t},{ }^{3} J=6.5 \mathrm{~Hz}\right), \mathrm{NH}$ protons were not assigned. ${ }^{13} \mathrm{C}$ NMR $\left(\mathrm{CDCl}_{3}, 297 \mathrm{~K}\right) \delta_{\mathrm{c}} \mathrm{ppm}: 28.8(4 \mathrm{C})$, 29.1 (2C), 42.7 (4C), 48.0-48.6 (8C, m), 111.3-111.7 (8C, m), 116.0 (4C), 129.3 (4C), 142.7 (4C), 148.7 (4C). 
7,10,13,16,19-Pentaazatricyclo[18.3.1.1 12,6]pentacosa1(24),2(25),3,5,20,22-hexaene, $3 \boldsymbol{h}$. Obtained from $94 \mathrm{mg}$ of pentaamine 2h. Eluent: $\mathrm{CH}_{2} \mathrm{Cl}_{2}-\mathrm{MeOH}-\mathrm{NH}_{3}$-aq 100:20:3. Yield $32 \mathrm{mg}$ (19\%). Pale-yellow glassy solid. $m / z$ (MALDI-TOF) found: 339.2373. $\mathrm{C}_{20} \mathrm{H}_{29} \mathrm{~N}_{5}$ requires $339.2423\left[\mathrm{M}^{+}\right] .{ }^{1} \mathrm{H}$ NMR $\left(\mathrm{CDCl}_{3}, 297\right.$ K) $\delta_{\mathrm{H}}$ ppm: $2.74\left(4 \mathrm{H}, \mathrm{t},{ }^{3} J=4.7 \mathrm{~Hz}\right), 2.83\left(4 \mathrm{H}, \mathrm{t},{ }^{3} J=4.8 \mathrm{~Hz}\right), 2.90$ $\left(4 \mathrm{H}, \mathrm{t},{ }^{3} J=6.1 \mathrm{~Hz}\right), 3.34\left(4 \mathrm{H}, \mathrm{t},{ }^{3} J=6.1 \mathrm{~Hz}\right), 6.60\left(2 \mathrm{H}, \mathrm{d},{ }^{3} J=7.3\right.$ $\mathrm{Hz}), 6.98(2 \mathrm{H}, \mathrm{s}), 6.99\left(2 \mathrm{H}, \mathrm{d},{ }^{3} J=6.7 \mathrm{~Hz}\right), 7.22\left(2 \mathrm{H}, \mathrm{t},{ }^{3} J=8.0 \mathrm{~Hz}\right)$, $\mathrm{NH}$ protons were not assigned. ${ }^{13} \mathrm{C} \mathrm{NMR}\left(\mathrm{CDCl}_{3}, 297 \mathrm{~K}\right) \delta_{\mathrm{c}} \mathrm{ppm}$ : 43.7 (2C), 48.6 (2C), 49.1 (2C), 49.5 (2C), 111.6 (2C), 112.8 (2C), $116.2(2 \mathrm{C}), 129.5(2 \mathrm{C}), 142.6(2 \mathrm{C}), 148.8(2 \mathrm{C})$.

$7,10,13,16,19,30,33,36,39,42-$ Decaazapentacyclo $\left[41.3 \cdot 1 \cdot 1^{2,6} \cdot 1^{20,24} \cdot 1^{25,29}\right]$ pentaconta-1(47), 2(50), 3, 5, 20(49), 21 , 23,25(48),26,28,43,45-dodecaene, $4 \boldsymbol{h}(\mathrm{n}=1)$. Obtained as byproduct in the synthesis of macrocycle 3g. Eluent: $\mathrm{CH}_{2} \mathrm{Cl}_{2}-\mathrm{MeOH}-$ $\mathrm{NH}_{3}$-aq 100:25:5. Yield $5 \mathrm{mg}$ (3\%). Pale-yellow glassy solid. $\mathrm{m} / \mathrm{z}$ (MALDI-TOF) found: $678.58 . \mathrm{C}_{40} \mathrm{H}_{58} \mathrm{~N}_{10}$ requires $678.48\left[\mathrm{M}^{+}\right] .{ }^{1} \mathrm{H}$ NMR $\left(\mathrm{CDCl}_{3}, 297 \mathrm{~K}\right) \delta_{\mathrm{H}}$ ppm: $2.73(16 \mathrm{H}$, br.s), 2.86 (8H, br.s), 3.22 (8H, br.s), $6.59\left(4 \mathrm{H}, \mathrm{d},{ }^{3} J=7.7 \mathrm{~Hz}\right), 6.82(4 \mathrm{H}$, br.s), $6.90(4 \mathrm{H}$, $\left.\mathrm{d},{ }^{3} \mathrm{~J}=6.7 \mathrm{~Hz}\right), 7.20\left(4 \mathrm{H}, \mathrm{t},{ }^{3} \mathrm{~J}=7.7 \mathrm{~Hz}\right), \mathrm{NH}$ protons were not assigned. ${ }^{13} \mathrm{C}$ NMR $\left(\mathrm{CDCl}_{3}, 297 \mathrm{~K}\right) \delta_{\mathrm{c}} \mathrm{ppm}: 43.5(4 \mathrm{C}), 48.6(4 \mathrm{C})$, 49.0 (4C), 49.2 (4C), 111.9 (8C), 116.5 (4C), 129.5 (4C), 142.8 (4C), 148.7 (4C).

10,13-Dioxa-7,16-diazatricyclo[15.3.1.1 1,6]docosa1(21),2(22),3,5,17,19-hexaene, 3i. Obtained from $74 \mathrm{mg}$ of dioxadiamine $2 \mathbf{i}$ in the presence of $\mathrm{Pd}(\mathrm{dba})_{2}(46 \mathrm{mg}, 16 \mathrm{~mol} \%)$ and BINAP (56 mg, 18 mol\%). Eluent: $\mathrm{CH}_{2} \mathrm{Cl}_{2}-\mathrm{MeOH}$ 100:1. Yield 60 mg (40\%). Pale-yellow crystals, m.p. $211-212^{\circ} \mathrm{C}$. $\mathrm{m} / \mathrm{z}$ (MALDITOF) found: 298.1681. $\mathrm{C}_{18} \mathrm{H}_{22} \mathrm{~N}_{2} \mathrm{O}_{2}$ requires $298.1706\left[\mathrm{M}^{+}\right] .{ }^{1} \mathrm{H}$ $\operatorname{NMR}\left(\mathrm{CDCl}_{3}, 297 \mathrm{~K}\right) \delta_{\mathrm{H}} \mathrm{ppm}: 3.49\left(4 \mathrm{H}, \mathrm{t},{ }^{3} \mathrm{~J}=5.5 \mathrm{~Hz}\right), 3.70(4 \mathrm{H}$, s), $3.71\left(4 \mathrm{H}, \mathrm{t},{ }^{3} J=5.6 \mathrm{~Hz}\right), 4.09(2 \mathrm{H}$, br.s $), 6.59\left(2 \mathrm{H}\right.$, ddd, ${ }^{3} J=8.0$ $\left.\mathrm{Hz},{ }^{4} J=1.5 \mathrm{~Hz},{ }^{4} J=0.8 \mathrm{~Hz}\right), 7.06\left(2 \mathrm{H}, \mathrm{d},{ }^{3} J=7.7 \mathrm{~Hz}\right), 7.19(2 \mathrm{H}, \mathrm{t}$, $\left.{ }^{3} J=7.7 \mathrm{~Hz}\right), 7.39\left(2 \mathrm{H}, \mathrm{t},{ }^{4} J=1.9 \mathrm{~Hz}\right) .{ }^{13} \mathrm{C} \mathrm{NMR}\left(\mathrm{CDCl}_{3}, 297 \mathrm{~K}\right) \delta_{\mathrm{c}}$ ppm: 45.2 (2C), 70.9 (2C), 72.6 (2C), 110.9 (2C), 114.2 (2C), 116.3 (2C), 128.9 (2C), 142.7 (2C), $148.9(2 \mathrm{C})$.

10,13,30,33-Tetraoxa-7,16,27,36-tetraazapentacyclo[35.3.1.1 $\left.1^{2,6} \cdot 1^{17,21} \cdot 1^{22,26}\right]$ tetratetraconta-1(41),2(44),3,5,17(43), 18,20,22(42),23,25,37,39-dodecaene, $4 \boldsymbol{i}(\mathrm{n}=1)$. Obtained as byproduct in the synthesis of macrocycle 3i. Eluent: $\mathrm{CH}_{2} \mathrm{Cl}_{2}-\mathrm{MeOH}$ $75: 1$. Yield $22 \mathrm{mg}(15 \%)$. Pale-yellow glassy solid. $\mathrm{m} / \mathrm{z}$ (MALDITOF) found: 596.3409. $\mathrm{C}_{36} \mathrm{H}_{44} \mathrm{~N}_{4} \mathrm{O}_{4}$ requires $596.3362\left[\mathrm{M}^{+}\right] .{ }^{1} \mathrm{H}$ $\operatorname{NMR}\left(\mathrm{CDCl}_{3}, 297 \mathrm{~K}\right) \delta_{\mathrm{H}}$ ppm: $3.34\left(8 \mathrm{H}, \mathrm{t},{ }^{3} \mathrm{~J}=4.7 \mathrm{~Hz}\right), 3.68(8 \mathrm{H}$, s), $3.73\left(8 \mathrm{H}, \mathrm{t},{ }^{3} J=4.8 \mathrm{~Hz}\right), 4.23(4 \mathrm{H}$, br.s $), 6.60\left(4 \mathrm{H}, \mathrm{d},{ }^{3} J=7.7\right.$ $\mathrm{Hz}), 6.79$ (4H, br.s), $6.91\left(4 \mathrm{H}, \mathrm{d},{ }^{3} J=7.1 \mathrm{~Hz}\right), 7.18\left(4 \mathrm{H}, \mathrm{t},{ }^{3} J=7.5\right.$ $\mathrm{Hz}) .{ }^{13} \mathrm{C} \mathrm{NMR}\left(\mathrm{CDCl}_{3}, 297 \mathrm{~K}\right) \delta_{\mathrm{c}} \mathrm{ppm}: 43.7$ (4C), $69.6(4 \mathrm{C}), 70.3$ (4C), 111.8 (4C), 112.5 (4C), 116.8 (4C), 129.5 (4C), 142.9 (4C), $148.5(4 \mathrm{C})$

$10,13,30,33,50,53$-Hexaoxa-7,16,27,36,47,56hexaazaheptacyclo $\left[55 \cdot 3 \cdot 1 \cdot 1^{2,6} \cdot 1^{17,21} \cdot 1^{22,26} \cdot 1^{37,41} \cdot 1^{42,46}\right]$ hexahexaconta-1(61),2(66), 3,5,17(65), 18,20,22(64), $23,25,37(63), 38,40,42(62), 43,45,57,59$-octadecaene, $4 i \quad(\mathrm{n}=2)$. Obtained as by-product in the synthesis of macrocycle 3i. Eluent: $\mathrm{CH}_{2} \mathrm{Cl}_{2}-\mathrm{MeOH}$ 20:1. Yield $24 \mathrm{mg}$ (16\%). Pale-yellow glassy solid. $m / z$ (MALDI-TOF) found: 894.56. $\mathrm{C}_{54} \mathrm{H}_{66} \mathrm{~N}_{6} \mathrm{O}_{6}$ requires 894.50 $\left[\mathrm{M}^{+}\right.$]. ${ }^{1} \mathrm{H}$ NMR $\left(\mathrm{CDCl}_{3}, 297 \mathrm{~K}\right) \delta_{\mathrm{H}}$ ppm: $3.36(12 \mathrm{H}$, br.s $), 3.67$ (12H, s), 3.72 (12H, br.s), 4.06 (6H, br.s), $6.62\left(6 \mathrm{H}, \mathrm{d},{ }^{3} J=7.5 \mathrm{~Hz}\right)$, $6.84(6 \mathrm{H}$, br.s $), 6.94\left(6 \mathrm{H}, \mathrm{d},{ }^{3} J=6.8 \mathrm{~Hz}\right), 7.22\left(6 \mathrm{H}, \mathrm{t},{ }^{3} J=7.4 \mathrm{~Hz}\right)$. ${ }^{13} \mathrm{C} \mathrm{NMR}\left(\mathrm{CDCl}_{3}, 297 \mathrm{~K}\right) \delta_{\mathrm{c}} \mathrm{ppm}: 43.6(6 \mathrm{C}), 69.7(6 \mathrm{C}), 70.3(6 \mathrm{C})$, 112.0 (6C), 112.1 (6C), 116.8 (6C), 129.5 (6C), 142.9 (6C), 148.5 (6C).

11,16-Dioxa-7,20-diazatricyclo[19.3.1.12,6]hexacosa1(25),2(26),3,5,21,23-hexaene, 3j. Obtained from $102 \mathrm{mg}$ of dioxadiamine 2j. Eluent: $\mathrm{CH}_{2} \mathrm{Cl}_{2}-\mathrm{MeOH} 100: 1$. Yield $78 \mathrm{mg}$ (44\%). Pale-yellow crystals, m.p. $111-112^{\circ} \mathrm{C} . \mathrm{m} / \mathrm{z}$ (MALDI-TOF) found: 354.2279. $\mathrm{C}_{22} \mathrm{H}_{30} \mathrm{~N}_{2} \mathrm{O}_{2}$ requires $354.2307\left[\mathrm{M}^{+}\right]$. ${ }^{1} \mathrm{H}$ NMR $\left(\mathrm{CDCl}_{3}\right.$, $297 \mathrm{~K}) \delta_{\mathrm{H}}$ ppm: $1.76\left(4 \mathrm{H}\right.$, quintet, $\left.{ }^{3} \mathrm{~J}=2.8 \mathrm{~Hz}\right), 1.90$ (4H, quintet, $\left.{ }^{3} J=5.8 \mathrm{~Hz}\right), 3.36\left(4 \mathrm{H}, \mathrm{t},{ }^{3} J=6.5 \mathrm{~Hz}\right), 3.47(4 \mathrm{H}, \mathrm{br} . \mathrm{s}), 3.61(4 \mathrm{H}, \mathrm{t}$, $\left.{ }^{3} J=5.3 \mathrm{~Hz}\right), 6.57\left(2 \mathrm{H}, \mathrm{ddd},{ }^{3} J=8.0 \mathrm{~Hz},{ }^{4} J=2.2 \mathrm{~Hz},{ }^{4} J=0.7 \mathrm{~Hz}\right)$, $6.99\left(2 \mathrm{H}, \mathrm{t},{ }^{4} J=2.1 \mathrm{~Hz}\right), 7.01\left(2 \mathrm{H}, \mathrm{d},{ }^{3} J=7.7 \mathrm{~Hz}\right), 7.24\left(2 \mathrm{H}, \mathrm{t},{ }^{3} J\right.$ $=7.8 \mathrm{~Hz}) \cdot{ }^{13} \mathrm{C} \mathrm{NMR}\left(\mathrm{CDCl}_{3}, 297 \mathrm{~K}\right) \delta_{\mathrm{c}} \mathrm{ppm}: 27.2(2 \mathrm{C}), 29.3(2 \mathrm{C})$, $43.2(2 \mathrm{C}), 70.3(2 \mathrm{C}), 71.2(2 \mathrm{C}), 111.5$ (4C), $115.8(2 \mathrm{C}), 129.3(2 \mathrm{C})$, $142.2(2 \mathrm{C}), 149.1(2 \mathrm{C})$.

11,16,35,40-Tetraoxa-7,20,31,44-tetraazapentacyclo[43.3.1.1 $\left.1^{2,6} \cdot 1^{21,25} \cdot 1^{26,30}\right]$ dopentaconta-1(49),2(52),3,5,21(51), $22,24,26(50), 27,29,45,47-d o d e c a e n e, 4 j(n=1)$. Obtained as byproduct in the synthesis of macrocycle 3j. Eluent: $\mathrm{CH}_{2} \mathrm{Cl}_{2}-\mathrm{MeOH}$ 75:1. Yield $20 \mathrm{mg}$ (12\%). Pale-yellow glassy solid. $\mathrm{m} / z$ (MALDITOF) found: 708.42. $\mathrm{C}_{44} \mathrm{H}_{60} \mathrm{~N}_{4} \mathrm{O}_{4}$ requires $708.46\left[\mathrm{M}^{+}\right] .{ }^{1} \mathrm{H}$ NMR $\left(\mathrm{CDCl}_{3}, 297 \mathrm{~K}\right) \delta_{\mathrm{H}} \mathrm{ppm}: 1.66\left(8 \mathrm{H}\right.$, quintet, $\left.{ }^{3} J=2.1 \mathrm{~Hz}\right), 1.85(8 \mathrm{H}$, quintet, $\left.{ }^{3} \mathrm{~J}=6.1 \mathrm{~Hz}\right), 3.23\left(8 \mathrm{H}, \mathrm{t},{ }^{3} \mathrm{~J}=6.4 \mathrm{~Hz}\right), 3.43\left(8 \mathrm{H}\right.$, quintet, ${ }^{3} \mathrm{~J}$ $=2.1 \mathrm{~Hz}), 3.52\left(8 \mathrm{H}, \mathrm{t},{ }^{3} \mathrm{~J}=5.7 \mathrm{~Hz}\right), 4.13(4 \mathrm{H}, \mathrm{br} . \mathrm{s}), 6.54\left(4 \mathrm{H}, \mathrm{dd},{ }^{3} \mathrm{~J}\right.$ $\left.=7.9 \mathrm{~Hz},{ }^{4} J=1.5 \mathrm{~Hz}\right), 6.77\left(4 \mathrm{H}, \mathrm{t},{ }^{4} J=1.8 \mathrm{~Hz}\right), 6.87\left(4 \mathrm{H}, \mathrm{d},{ }^{3} J=\right.$ $7.6 \mathrm{~Hz}), 7.18\left(4 \mathrm{H}, \mathrm{t},{ }^{3} J=7.8 \mathrm{~Hz}\right) .{ }^{13} \mathrm{C}$ NMR $\left(\mathrm{CDCl}_{3}, 297 \mathrm{~K}\right) \delta_{\mathrm{c}} \mathrm{ppm}:$ 26.6 (4C), 29.3 (4C), 42.2 (4C), 69.5 (4C), 70.8 (4C), 111.4 (4C), 111.7 (4C), 116.3 (4C), 129.3 (4C), 143.0 (4C), 148.8 (4C).

11,14,17-Trioxa-7,21-diazatricyclo[20.3.1.12,6]heptacosa1(26),2(27),3,5,22,24-hexaene, $3 \boldsymbol{k}$. Obtained from $110 \mathrm{mg}$ of trioxadiamine 2k. Eluent $\mathrm{CH}_{2} \mathrm{Cl}_{2}-\mathrm{MeOH}$ 100:1. Yield $71 \mathrm{mg}$ (38\%). Pale-yellow crystals, m.p. $74-75^{\circ} \mathrm{C} . \mathrm{m} / \mathrm{z}$ (MALDI-TOF) found: $370.2240 . \mathrm{C}_{22} \mathrm{H}_{30} \mathrm{~N}_{2} \mathrm{O}_{3}$ requires $370.2256\left[\mathrm{M}^{+}\right] . \delta_{\mathrm{H}}\left(\mathrm{CDCl}_{3}\right.$, $297 \mathrm{~K}) 1.91\left(4 \mathrm{H}\right.$, quintet, $\left.{ }^{3} \mathrm{~J}=5.9 \mathrm{~Hz}\right), 3.35\left(4 \mathrm{H}, \mathrm{t},{ }^{3} J=6.5 \mathrm{~Hz}\right)$, 3.60-3.66 (8H, m), 3.73-3.77 (4H, m), 4.26 (2H, br.s), 6.57 (2H, d, $\left.{ }^{3} J=7.9 \mathrm{~Hz}\right), 6.94$ (2H, br.s), $6.97\left(2 \mathrm{H}, \mathrm{d},{ }^{3} J=7.7 \mathrm{~Hz}\right), 7.21\left(2 \mathrm{H}, \mathrm{t},{ }^{3} J\right.$ $=7.8 \mathrm{~Hz}) \cdot{ }^{13} \mathrm{C} \mathrm{NMR}\left(\mathrm{CDCl}_{3}, 297 \mathrm{~K}\right) \delta_{\mathrm{c}} \mathrm{ppm}: 29.3(2 \mathrm{C}), 42.5(2 \mathrm{C})$, 70.1 (2C), 70.7 (2C), $71.0(2 \mathrm{C}), 111.1$ (2C), 111.9 (2C), 115.9 (2C), $129.2(2 \mathrm{C}), 142.5(2 \mathrm{C}), 149.0(2 \mathrm{C})$.

11,14,17,36,39,42-Hexaoxa-7,21,32,46-tetraazapentacyclo[45.3.1.1 $\left.1^{2,6} \cdot 1^{22,26} \cdot 1^{27,31}\right]$ tetrapentaconta-1(51), 2(54), $3,5,22(53), 23,25,27(52), 28,30,47,49$-dodecaene, $4 \boldsymbol{k}(\mathrm{n}=1)$. Obtained as by-product in the synthesis of macrocycle 3k. Eluent: $\mathrm{CH}_{2} \mathrm{Cl}_{2}-$ $\mathrm{MeOH}$ 50:1. Yield $22 \mathrm{mg}$ (12\%). Pale-yellow glassy solid. $\mathrm{m} / \mathrm{z}$ (MALDI-TOF) found: 740.4473. $\mathrm{C}_{44} \mathrm{H}_{60} \mathrm{~N}_{4} \mathrm{O}_{6}$ requires 740.4512 $\left[\mathrm{M}^{+}\right] .{ }^{1} \mathrm{H}$ NMR $\left(\mathrm{CDCl}_{3}, 297 \mathrm{~K}\right) \delta_{\mathrm{H}} \mathrm{ppm}: 1.84\left(8 \mathrm{H}\right.$, quintet, ${ }^{3} J=6.0$ $\mathrm{Hz}), 3.23\left(8 \mathrm{H}, \mathrm{t},{ }^{3} \mathrm{~J}=6.4 \mathrm{~Hz}\right), 3.55\left(8 \mathrm{H}, \mathrm{t},{ }^{3} \mathrm{~J}=5.8 \mathrm{~Hz}\right), 3.55-3.60(8 \mathrm{H}$, m), 3.63-3.67 (8H, m), 4.18 (4H, br.s), $6.54\left(4 \mathrm{H}, \mathrm{dd},{ }^{3} \mathrm{~J}=7.7 \mathrm{~Hz},{ }^{4} \mathrm{~J}\right.$ $=1.9 \mathrm{~Hz}), 6.73\left(4 \mathrm{H}\right.$, br.s), $6.85\left(4 \mathrm{H}, \mathrm{d},{ }^{3} J=7.4 \mathrm{~Hz}\right), 7.17\left(4 \mathrm{H}, \mathrm{t},{ }^{3} J=\right.$ $8.0 \mathrm{~Hz}) .{ }^{13} \mathrm{C} \mathrm{NMR}\left(\mathrm{CDCl}_{3}, 297 \mathrm{~K}\right) \delta_{\mathrm{c}} \mathrm{ppm}: 29.0(4 \mathrm{C}), 41.7(4 \mathrm{C}), 69.7$ (4C), 70.2 (4C), 70.6 (4C), 111.3 (4C), 111.8 (4C), 116.1 (4C), 129.3 (4C), 143.0 (4C), 148.8 (4C).

Synthesis of $N, N^{\prime}-\left(2,2^{\prime}\right.$-(ethane-1,2-diylbis(oxy))bis(ethane2,1-diyl))bis(5'-bromobiphenyl-3-amine), $5 \boldsymbol{i}$, is analogous to the procedure described for the preparation of macrocycles $\mathbf{3}$, from 3,3'-dibromobiphenyl (1) (1.5 mmol, $468 \mathrm{mg})$ and dioxadiamine $2 \mathbf{i}(0.5 \mathrm{mmol}, 74 \mathrm{mg})$, in absolute dioxane $(5 \mathrm{ml})$, in the presence of $\mathrm{Pd}(\mathrm{dba})_{2}(12 \mathrm{mg}, 4 \mathrm{~mol} \%)$, Xanthphos (13 mg, $5 \mathrm{~mol} \%$ ) and ${ }^{t} \mathrm{BuONa}(1.5 \mathrm{mmol})$. Reflux time: $8 \mathrm{~h}$. Eluent; $\mathrm{CH}_{2} \mathrm{Cl}_{2}-\mathrm{MeOH}$ 200:1. Yield $82 \mathrm{mg}$ (27\%). Pale-yellow glassy solid. $\mathrm{m} / \mathrm{z}$ (MALDITOF) found: 608.0713. $\mathrm{C}_{30} \mathrm{H}_{30} \mathrm{Br}_{2} \mathrm{~N}_{2} \mathrm{O}_{2}$ requires $608.0674\left[\mathrm{M}^{+}\right] .{ }^{1} \mathrm{H}$ NMR $\left(\mathrm{CDCl}_{3}, 297 \mathrm{~K}\right) \delta_{\mathrm{H}} \mathrm{ppm:} 3.36\left(4 \mathrm{H}, \mathrm{t},{ }^{3} J=5.2 \mathrm{~Hz}\right), 3.68(4 \mathrm{H}, \mathrm{s})$, $3.74\left(4 \mathrm{H}, \mathrm{t},{ }^{3} \mathrm{~J}=5.2 \mathrm{~Hz}\right), 4.20(2 \mathrm{H}, \mathrm{br} . \mathrm{s}), 6.63\left(2 \mathrm{H}, \mathrm{dd},{ }^{3} J=8.1 \mathrm{~Hz}\right.$, $\left.{ }^{4} J=1.4 \mathrm{~Hz}\right), 6.77\left(2 \mathrm{H}, \mathrm{t},{ }^{4} J=1.4 \mathrm{~Hz}\right), 6.88\left(2 \mathrm{H}, \mathrm{d},{ }^{3} J=7.7 \mathrm{~Hz}\right), 7.22$ $\left(2 \mathrm{H}, \mathrm{t},{ }^{3} J=7.9 \mathrm{~Hz}\right), 7.25\left(2 \mathrm{H}, \mathrm{d},{ }^{3} J=7.8 \mathrm{~Hz}\right), 7.42-7.47(4 \mathrm{H}, \mathrm{m}), 7.69$ $\left(2 \mathrm{H}, \mathrm{t},{ }^{4} J=1.6 \mathrm{~Hz}\right) .{ }^{13} \mathrm{C} \mathrm{NMR}\left(\mathrm{CDCl}_{3}, 297 \mathrm{~K}\right) \delta_{\mathrm{c}} \mathrm{ppm}: 43.5(2 \mathrm{C})$, $69.6(2 \mathrm{C}), 70.2(2 \mathrm{C}), 111.6(2 \mathrm{C}), 112.6(2 \mathrm{C}), 116.6(2 \mathrm{C}), 122.7(2 \mathrm{C})$, 125.7 (2C), 129.7 (2C), 129.9 (2C), 130.0 (2C), 130.1 (2C), 140.8 (2C), 143.8 (2C), 148.6 (2C).

Synthesis of $N^{\beta}, N^{3}$-bis(2-(2-(2-aminoethoxy)ethoxy)ethyl) biphenyl-3,3'-diamine, $\mathbf{6 i}$, is analogous to the procedure described for the preparation of compound $\mathbf{5 i}$, from 3,3'-dibromobiphenyl $\mathbf{1}$ $(0.5 \mathrm{mmol}, 156 \mathrm{mg})$ and dioxadiamine $2 \mathbf{i}(2 \mathrm{mmol}, 296 \mathrm{mg})$, in absolute dioxane $(5 \mathrm{ml})$, in the presence of $\mathrm{Pd}(\mathrm{dba})_{2}(12 \mathrm{mg}, 4$ mol\%), BINAP (14 mg, $4.5 \mathrm{~mol} \%$ ) and ${ }^{t} \mathrm{BuONa}(1.5 \mathrm{mmol})$. Reflux time: 8 h. $m / z$ (MALDI-TOF) found: $446.42 . \mathrm{C}_{24} \mathrm{H}_{38} \mathrm{~N}_{4} \mathrm{O}_{4}$ requires $446.29\left[\mathrm{M}^{+}\right] .{ }^{1} \mathrm{H}$ NMR $\left(\mathrm{CDCl}_{3}, 297 \mathrm{~K}\right) \delta_{\mathrm{H}} \mathrm{ppm}: 2.83\left(4 \mathrm{H}, \mathrm{t},{ }^{3} J=\right.$ 
$5.3 \mathrm{~Hz}), 3.32\left(4 \mathrm{H}, \mathrm{t},{ }^{3} J=5.3 \mathrm{~Hz}\right), 3.49\left(4 \mathrm{H}, \mathrm{t},{ }^{3} J=5.2 \mathrm{~Hz}\right), 3.60-$ $3.66(8 \mathrm{H}, \mathrm{m}), 3.71\left(4 \mathrm{H}, \mathrm{t},{ }^{3} J=5.2 \mathrm{~Hz}\right), 6.59\left(2 \mathrm{H}, \mathrm{ddd},{ }^{3} J=8.1 \mathrm{~Hz}\right.$, $\left.{ }^{4} J=2.4 \mathrm{~Hz},{ }^{4} J=0.7 \mathrm{~Hz}\right), 6.80\left(2 \mathrm{H}, \mathrm{t},{ }^{4} J=2.1 \mathrm{~Hz}\right), 6.89\left(2 \mathrm{H}, \mathrm{d},{ }^{3} J\right.$ $=7.6 \mathrm{~Hz}), 7.19\left(2 \mathrm{H}, \mathrm{t},{ }^{3} \mathrm{~J}=7.8 \mathrm{~Hz}\right), \mathrm{NH}$ protons were not assigned. ${ }^{13} \mathrm{C} \mathrm{NMR}\left(\mathrm{CDCl}_{3}, 297 \mathrm{~K}\right) \delta_{\mathrm{c}} \mathrm{ppm}: 41.5(2 \mathrm{C}), 43.4(2 \mathrm{C}), 69.6(2 \mathrm{C})$, 70.2 (4C), 73.4 (2C), 112.0 (4C), 116.7 (2C), 129.3 (2C), 142.8 (2C), $148.4(2 \mathrm{C})$.

Synthesis of cyclodimer $4 \mathbf{i}(n=1)$ from compound $\mathbf{5 i}$ is analogous to the procedure described for the preparation of macrocycles 3, from compound $\mathbf{5 i}(0.135 \mathrm{mmol}, 82 \mathrm{mg})$ and dioxadiamine $2 \mathbf{i}(0.135 \mathrm{mmol}, 20 \mathrm{mg})$, in absolute dioxane $(7 \mathrm{ml})$, in the presence of $\mathrm{Pd}(\mathrm{dba})_{2}(6.5 \mathrm{mg}, 8 \mathrm{~mol} \%)$, BINAP $(7.5 \mathrm{mg}, 9$ $\mathrm{mol} \%)$ and ${ }^{t} \mathrm{BuONa}(0.4 \mathrm{mmol}, 39 \mathrm{mg})$. Reflux time: $24 \mathrm{~h}$. Eluent; $\mathrm{CH}_{2} \mathrm{Cl}_{2}-\mathrm{MeOH}$ 20:1. Yield $33 \mathrm{mg}(27 \%)$.

Synthesis of cyclodimer $4 \mathbf{i}(n=1)$ from compound $\mathbf{6 i}$ is analogous to the procedure described for the preparation of macrocycles $\mathbf{3}$, from the mixture of compound $\mathbf{6 i}$ ( $\mathrm{ca} 0.25 \mathrm{mmol}$ ) with dioxadiamine $2 \mathbf{i}$ (ca $0.5 \mathrm{mmol}$ ) and 3,3'-dibromobiphenyl (1) $(0.75 \mathrm{mmol}, 234 \mathrm{mg})$, in absolute dioxane $(12 \mathrm{ml})$, in the presence of $\mathrm{Pd}(\mathrm{dba})_{2}$ (36 mg, $8 \mathrm{~mol} \%$ ), BINAP (42 mg, $9 \mathrm{~mol} \%$ ) and ${ }^{t} \mathrm{BuONa}$ (2.25 mmol, $216 \mathrm{mg}$ ). Reflux time: $24 \mathrm{~h}$. Eluent; $\mathrm{CH}_{2} \mathrm{Cl}_{2}-\mathrm{MeOH}$ 20:1. Yield $98 \mathrm{mg}(44 \%)$.

\section{Results and Discussion}

The reactions of equimolar amounts of 3,3'-dibromobiphenyl $\mathbf{1}$ with a variety of di- and polyamines 2a-k (Figure 1) were run in enough dilute dioxane solutions $(\mathrm{c}=0.02 \mathrm{M})$ using $\mathrm{Pd}(\mathrm{dba})_{2} / \mathrm{BINAP}(8 / 9 \mathrm{~mol} \%)$ catalytic system $^{[24]}$ which was found to be almost universal for the synthesis of polyazamacrocycles, the products were isolated by column chromatography on silica gel. The results are given in Table 1.

As expected, propane-1,3-diamine $\mathbf{2 a}$ was too short to give a desired mono-cycle $\mathbf{3}$, and it produced cyclodimer $4 \mathbf{a}(\mathrm{n}=1)$ in $24 \%$ yield as well as a mixture of higher mass oligomers 4a $(n=2-7)(37 \%)$ (Table 1, entry 1). Diethylenetriamine $\mathbf{2 b}$ (7 atoms in the chain) also gave only cyclooligomers $\mathbf{4 b}(\mathrm{n}=1-5)$ (entry 2$)$, but beginning from triamine 2c (9 atoms) target macrocycles 6 were formed successfully in yields from moderate to good (entries 3-13). The best yields (44\%, entries 6, 11) were achieved with tetraamine $\mathbf{2 f}$ and dioxadiamine $\mathbf{2} \mathbf{j}$, also enough high yields for the macrocyclization reaction ( $c a$ $40 \%$ ) were afforded by tetraamine $\mathbf{2 g}$ and trioxadiamine $\mathbf{2 k}$ (entries 7, 12). We increased the yield of the macrocycle $3 \mathbf{k}$ to $45 \%$ by the application of 2-dicyclohexylphosphino2'-dimethylamino-biphenyl (DavePHOS) ligand instead of BINAP (entry 13), however, this approach did not work in the case of dioxadiamine $2 \mathbf{i}$, but the use of $16 \%$ mol catalyst gave corresponding macrocycle $\mathbf{3 i}$ in $40 \%$ yield (entry 10). In many cases cyclodimers $4(n=1)$ were isolated from oligomeric mixtures in $8-25 \%$ yields, and in the reactions with dioxadiamine $2 \mathbf{i}$ even cyclotrimer $\mathbf{4 i}(n=2)$ was obtained separately in $16 \%$ yield. It is to be mentioned that $40 \%$ yields of polyazamacrocycles are among the highest ever achieved by the Pd-catalyzed amination of dihaloarenes, e.g. they notably surpass those obtained recently with 2,7-dibromonaphthalene. ${ }^{[25]}$ Mono-cycles 3 contain from 15 (3c) to 21 atoms $(\mathbf{3 k})$ in the cycle, cyclodimers 4 possess from $22(\mathbf{4 a}, \mathrm{n}=1)$ to 42 atoms $(\mathbf{4 k}, \mathrm{n}=1)$, while cyclotrimer $\mathbf{4 i}$ $(n=2)$ has a cavity formed by 48 atoms.

Cyclodimers which possess two biphenyl units and two polyamine chains and thus have a larger cavity size are of interest for the coordination studies with big cations and anions and organic molecules. We elaborated two approaches to such compounds. According to the first route, we synthesized $N, N$ 'di(bromobiphenyl) substituted dioxadiamine 5i which was<smiles>Brc1cccc(-c2cccc(Br)c2)c1</smiles>

1

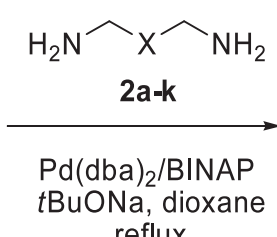

reflux<smiles>[X]CNc1cccc(-c2cccc(NCC)c2)c1</smiles>

$3 \mathbf{c - k}, 16-45 \%$

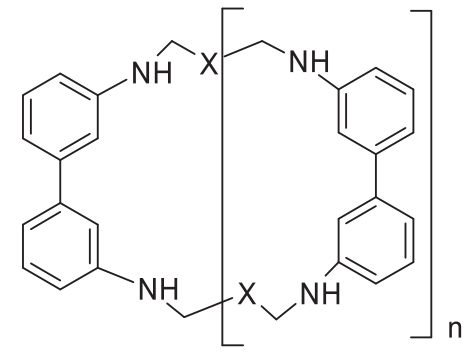

4a-k, $18-61 \%$<smiles>NCCCNCCCN</smiles><smiles>NCCNCCNCCCNCCNCCN</smiles><smiles>NCCCNCCNCCCN</smiles><smiles>NCCCNCCCNCCCN</smiles><smiles>NCCNCCNCCNCCN</smiles><smiles>NCCOCCOCCN</smiles>
2i<smiles>NCCCOCCCCOCCCN</smiles><smiles>NCCCOCCOCCOCCCN</smiles>

Figure 1. 
Table 1. Pd-Catalyzed amination of 3,3'-dibromobiphenyl (1).

\begin{tabular}{|c|c|c|c|c|}
\hline Entry & Amine & $\mathrm{Pd}(\mathrm{dba})_{2} / \mathrm{BINAP}, \mathrm{mol} \%$ & Isolated yields of $\mathbf{3}, \%$ & Yields of $4, \%$ \\
\hline 1 & $2 a$ & $8 / 9$ & 3a, 0 & $\mathbf{4 a}(\mathrm{n}=1), 24$ \\
\hline 2 & $2 b$ & $8 / 9$ & $\mathbf{3 b}, 0$ & $\begin{array}{l}\mathbf{4 a}(\mathrm{n}=2-7), 37 \text { (mixture) } \\
\mathbf{4 b}(\mathrm{n}=1), 23 \\
\mathbf{4 b}(\mathrm{n}=2-5), 38 \text { (mixture) }\end{array}$ \\
\hline 3 & $2 c$ & $8 / 9$ & $3 c, 25$ & $\begin{array}{l}\mathbf{4 c}(\mathrm{n}=1), 21 \\
\mathbf{4 c}(\mathrm{n}=2-5), 23 \text { (mixture) }\end{array}$ \\
\hline 4 & 2d & $8 / 9$ & 3d, 16 & $\mathbf{4 d}(\mathrm{n}=1,2), 18$ (mixture) \\
\hline 5 & $2 \mathrm{e}$ & $8 / 9$ & 3e, 26 & $\begin{array}{l}4 \mathbf{e}(n=1), 8 \\
4 \mathbf{e}(\mathrm{n}=1-3), 27 \text { (mixture) }\end{array}$ \\
\hline 6 & $2 \mathrm{f}$ & $8 / 9$ & 3f, 44 & $\begin{array}{l}\mathbf{4 f}(\mathrm{n}=1), 25 \\
\mathbf{4 f}(\mathrm{n}=1,2), 17 \text { (mixture) }\end{array}$ \\
\hline 7 & $2 \mathrm{~g}$ & $8 / 9$ & $3 g, 41$ & $\mathbf{4 g}(\mathrm{n}=1-3), 19$ (mixture) \\
\hline 8 & $2 \mathrm{~h}$ & $8 / 9$ & 3h, 19 & $\begin{array}{l}\mathbf{4 h}(\mathrm{n}=1), 3 \\
\mathbf{4 h}(\mathrm{n}=1,2), 12 \text { (mixture) }\end{array}$ \\
\hline 9 & $2 \mathbf{i}$ & $8 / 9$ & $3 \mathbf{i}, 19$ & $4 \mathbf{i}(\mathrm{n}=1), 12$ \\
\hline 10 & $2 \mathbf{i}$ & $16 / 18$ & $3 \mathbf{i}, 40$ & $\begin{array}{l}4 \mathbf{i}(\mathrm{n}=1), 15 \\
\mathbf{4 i}(\mathrm{n}=2), 16\end{array}$ \\
\hline 11 & $2 j$ & $8 / 9$ & $3 \mathbf{j}, 44$ & $\begin{array}{l}\mathbf{4 j}(\mathrm{n}=1), 12 \\
\mathbf{4 j}(\mathrm{n}=1-4), 18 \text { (mixture) }\end{array}$ \\
\hline 12 & $2 k$ & $8 / 9$ & $3 \mathbf{k}, 38$ & $\begin{array}{l}\mathbf{4 k}(\mathrm{n}=1), 12 \\
\mathbf{4 k}(\mathrm{n}=1-4), 17 \text { (mixture) }\end{array}$ \\
\hline $13^{\text {a) }}$ & $2 k$ & $8 / 10$ & $3 \mathbf{k}, 45$ & $\begin{array}{l}\mathbf{4 k}(\mathrm{n}=1), 21 \\
\mathbf{4 k}(\mathrm{n}=1-3), 9 \text { (mixture) }\end{array}$ \\
\hline
\end{tabular}

a) DavePHOS was used instead of BINAP

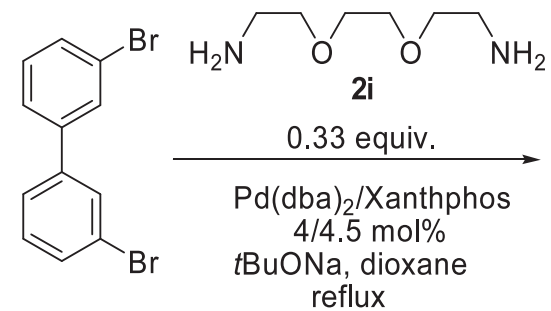

reflux

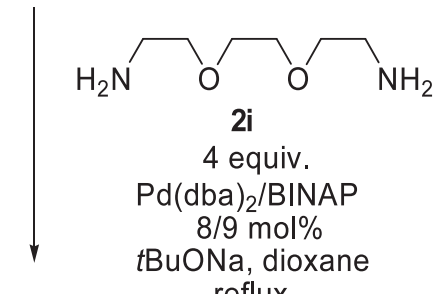<smiles>Brc1cccc(NCCOCCOCCNc2cccc(-c3cccc(Br)c3)c2)c1</smiles>

5i, $27 \%$<smiles>NCCOCCOCCN</smiles>

$\mathrm{Pd}(\mathrm{dba})_{2} / \mathrm{BINAP}$ $8 / 9 \mathrm{~mol} \%$ tBuONa, dioxane reflux
$4 i, 27 \%$

Figure 2.

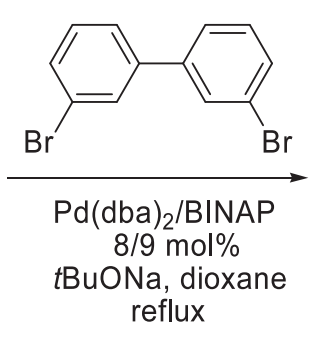

$4 i, 44 \%$

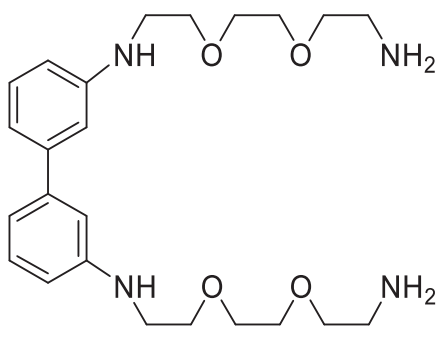

6i in situ<smiles>c1cc2cc(c1)NCCOCCCNc1cccc(c1)-c1cccc(c1)NCCOCCOCCCN2</smiles> 
taken as an exemplary amine (Figure 2). Three equivalents of 3,3'-dibromobiphenyl 1 were employed in the presence of $4 \mathrm{~mol} \%$ catalyst to give $\mathbf{5 i}$ in $27 \%$ yield. The reaction was severely complicated by $N, N$-diarylation and diamination processes, thus the application of Xanthphos ligand instead of BINAP was helpful. The cyclization into cyclodimers with dioxadiamine $2 \mathbf{i}$ was carried out under the conditions similar to those used for the macrocycles 3 . As a result, $4 \mathbf{i}(n=1)$ was isolated in $27 \%$ yield. The attempts to introduce diaryl derivative 5i in situ were totally unsuccessful. Alternative approach included the synthesis of bis(dioxadiamine) substituted biphenyl 6i using 4 equivalents of dioxadiamine 2i. The yield of this compounds in the reaction mixtures was very high according to NMR data, and $\mathbf{6 i}$ was used in situ in the further reaction with 3,3'-dibromobiphenyl (Scheme 2). Target macrocycle $4 \mathbf{i}(\mathrm{n}=1)$ was obtained in $44 \%$ yield. This approach to cyclodimers was found to be much more efficient because it is carried out as a one-pot procedure and provides higher overall yield of the target compound.

\section{Conclusions}

To sum up, we have investigated 3,3'-dibromobiphenyl in the Pd-catalyzed amination reactions with polyamines and oxadiamines, demonstrated the possibilitites to obtain polyazamacrocycles in enough high yields, elaborated two approaches to the corresponding cyclodimers and found out the advantage of the route through bis(polyamine) derivatives.

Acknowledgements. This work was supported by RFBR grants 09-03-00735, 08-03-00628 and by the Russian Academy of Sciences program P-8 "Development of the methods for the synthesis of new chemicals and creation of new materials".

\section{References}

1. Kohama H., Yoshinaga M., Ishizu K. Bull. Chem. Soc. Jpn. 1980, 53, 3707-3708.

2. Reinhoudt D.N., de Jong F., van de Vondervoort E.M. Tetrahedron 1981, 37, 1753-1762.
3. Reinhoudt D.N., de Jong F., van de Vondervoort E.M. Tetrahedron 1981, 37, 1985-1990.

4. Rebek Jr. J. Acc. Chem. Res. 1984, 17, 258-264.

5. Rebek Jr.J. Costello T., Marshall L., Wattley R., Gadwood R.C., Onan K. J. Am. Chem. Soc. 1985, 107, 7481-7487.

6. Rebek Jr.J., Wattley R.V., Costello T., Gadwood R., Marshall L. J. Am. Chem. Soc. 1980, 102, 7398-7400.

7. Gaviña F., Luis S.V., Costero A.M., Burguete M.I., Rebek Jr., J. J. Am. Chem. Soc. 1988, 110, 7140-7143.

8. Burguete M.I., Diaz P., Garcia-España E., Luis S.V., Miravet J.F., Querol M., Ramirez J.A. Chem. Commun. 1999, 649650.

9. Pearson D.P.J., Leigh S.J., Sutherland I.O. J. Chem. Soc., Perkin Trans. 1979, 1, 3113-3126.

10. Montero A., Albericio F., Royo M., Herradón B. Eur. J. Org. Chem. 2007, 1301-1308.

11. Artz S.P., Cram D.J. J. Am. Chem. Soc. 1984, 106, 2160-2171.

12. Choi K., Hamilton A.D. J. Am. Chem. Soc. 2003, 125, 1024110249.

13. Kuhnert N., Straßnig K., Lopez-Periago A.M. Tetrahedron: Asymmetry 2002, 13, 123-128.

14. Kuhnert N., Rossignolo G.M., Lopez-Periago A.M. Org. Biomol. Chem., 2003, 1, 1157-1170.

15. Patel H.K., Kilburn J.D., Langley G.J., Edwards P.D., Mitchell T., Southgate R. Tetrahedron Lett. 1994, 35, 481-484.

16. Lloyd-Williams P., Giralt E. Chem. Soc. Rev. 2001, 30, 145157.

17. Yang B.H., Buchwald S.L. J. Organomet. Chem. 1999, 576, 125-146.

18. Beletskaya I.P., Bessmertnykh A.G., Averin A.D., Denat F., Guilard R. Eur. J. Org. Chem. 2005, 281-305.

19. Averin A.D., Ulanovskaya O.A., Fedotenko I.A., Borisenko A.A., Serebryakova M.V., Beletskaya I.P. Helv. Chim. Acta 2005, 88, 1983-2002.

20. Averin A.D., Ulanovskaya O.A., Pleshkova N.A., Borisenko A.A., Beletskaya I.P. Coll. Czech. Chem. Comm. 2007, 72, 785-819.

21. Averin A.D., Shukhaev A.V., Golub S.L., Buryak A.K., Beletskaya I.P. Synthesis 2007, 2995-3012.

22. Ukai T., Kawazura H., Ishii Y., Bonnet J.J., Ibers J.A. J. Organomet. Chem. 1974, 65, 253-266.

23. Snyder H.R., Weaver C., Marshall C.D. J. Am. Chem. Soc. 1949, 71, 289-291.

24. Wolfe J.P., Buchwald S.L. J. Org. Chem. 2000, 65, 1147-1157.

25. Averin A.D., Uglov A. N., Beletskaya I.P. Chem. Lett. 2008, 37, 1074-1075. 